\title{
Superfluid-Insulator Transition and Fermion Pairing in Bose-Fermi Mixtures
}

\author{
Kun Yang \\ NHMFL and Department of Physics, Florida State University, Tallahassee, Florida 32306, USA and \\ Zhejiang Institute of Modern Physics, Zhejiang University, Hangzhou 310027, P.R. China
}

(Dated: November 30, 2018)

\begin{abstract}
It is well known that bosons on an optical lattice undergo a second-order superfluid-insulator transition (SIT) when the lattice potential increases. In this paper we study SIT when fermions coexist with the bosons. We find that the critical properties of particle-hole symmetric SIT with dynamical exponent $z=1$ is modified when fermions are present; it either becomes a fluctuationdriven first order transition or a different second-order transition. On the other hand the more generic particle-hole asymmetric (with $z=2$ ) SIT is stable against coupling with fermions. We also discuss pairing interaction between fermions mediated by quantum critical fluctuations near SIT, and show that the fermion pairing/superfluid transition temperature peaks near SIT when a single species of fermion is present.
\end{abstract}

\section{INTRODUCTION}

The observation of superfluid-insulator transition (SIT) of bosons on optical lattices [1, 2] is arguably one of the most important recent developments in the field of cold atom physics; it triggered a tremendous amount of interest and activities in studying strong correlation physics using optical lattices. More recently, much work has focused on strong interaction/correlation physics that involve fermions, like superfluidity resulting from fermion pairing, and realization of various exotic phases proposed in the context of electronic condensed matter physics 2]. Another direction of thrust is BoseFermi mixtures on optical lattices; these are new manybody systems that have no direct analogy in electronic condensed matter systems.

In this paper we consider boson SIT on optical lattices in Bose-Fermi mixtures in two- and threedimensions, and consider two separate situations: (i) Bosonic atoms coexist with fermionic atoms. (ii) The bosons are molecules made of two fermionic atoms of different species (or opposite "spins"), and the numbers of fermions are different for different spins. Due to the imbalance some of the fermions are unpaired and coexist with bosonic molecules. While the physical settings are very different, we show that the effective field theory describing the SIT turn out to be the same for both cases. We find that the presence of fermion do not affect the critical properties of SIT for most parts of the phase boundary separating the superfluid and insulating phases, provided the Bose-Fermi interaction is sufficiently weak. On the other hand near some special point with (emerging) particle-hole symmetry, fermions do affect the critical properties of SIT. These are the subjects of section II below.

Bosons mediate an attractive interaction between fermions, which leads to fermion pairing and superfluidity at low temperature. In section III we discuss this pairing interaction near boson SIT, which is dominated by critical fluctuations. We show that under certain condi- tions the fermion superfluid transition temperature peaks near SIT.

A few concluding remarks are offered in section IV.

\section{BOSON SUPERFLUID-INSULATOR TRANSITION}

Theoretically, the SIT is well understood in purely bosonic systems based on simple models like the BoseHubbard model [3, 4]:

$$
H_{B}=-t_{b} \sum_{<i j>} b_{i}^{\dagger} b_{j}-\mu_{b} \sum_{i} n_{b i}+\frac{U}{2} \sum_{i} n_{b i}\left(n_{b i}-1\right),
$$

where $b_{i}$ is the boson annihilation operator on site $i$, $n_{b i}=b_{i}^{\dagger} b_{i}, U>0$ is the on-site repulsion between the bosons, $t_{b}$ is the boson hopping matrix element and $\mu_{b}$ is boson chemical potential. The schematic phase diagram of (1) at zero temperature $(T=0)$ can be found in Fig. 1a, where there are two phases: At small $t_{b} / U$ the system is a Mott insulator with an integer number of bosons per site; for larger $t_{b} / U$ bosons declocalize and Bose condense, resulting in a superfluid phase. The superfluidinsulator transition (SIT) is continuous, and depending on the value of boson chemical potential $\mu_{b}$ there are two universality classes for the SIT [4]: (a) Generically, either particles or holes condense at the SIT, and the transition is the dilute Bose gas transition with dynamical exponent $z=2$; (b) at the tips of the lobes of the Mott insulator phase, particles and holes condense simultaneously, and the SIT is in the universality class of $d+1$ dimensional $X Y$ transition with $z=1$ (here $d$ is the spatial dimension of the system). The dynamical exponent $z$ is the critical exponent that characterizes the relation between energy/freqeuncy and momentum/wavevector of quantum critical excitations/fluctuations: $\omega_{k} \sim k^{z}$. For the two universality classes of SIT here the values of $z$ can be understood from the following considerations: (a) For the particle-hole asymmetric case, the critical excitations are either particles or holes that become gapless 


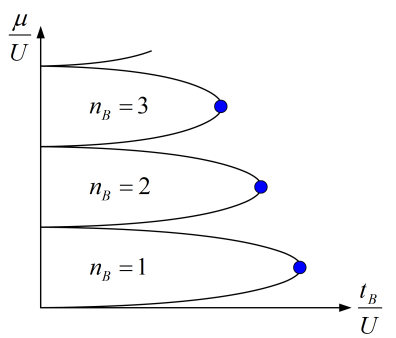

(a)

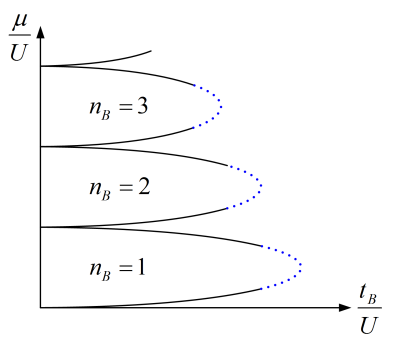

(b)
FIG. 1: (Color on-line) (a) Schematic phase diagram of Bose Hubbard model. Solid lines are 2nd order phase boundaries separating superfluid phase from Mott insulator phases with fixed boson number $n_{B}$ per site. For each lobe of the Mott insulator characterized by a fixed $n_{B}$, the superfluid-insulator transition (SIT) is triggered by the condensation of either particles (upper half of the lobe) or holes (lower half of the lobe), except at the tip of the lobe (blue dots) where particles and holes condense simultaneously. (b) In the presence of fermions, SIT may become 1st order near the tips of MI lobes (blue dotted lines).

and start to condense at SIT; they have non-relativistic dispersion relation $\omega_{k}=k^{2} /\left(2 m^{*}\right)\left(m^{*}\right.$ is their effective mass), and $z=2$ simply reflects this non-relativistic dispersion. (b) In the particle-hole symmetric case, both particles and holes become gapless at SIT, and must be treated on equal-footing. Thus the effective field theory contains both particle and antiparticle (hole), which turn out to be a relativistic quantum field theory with emergent Lorentz symmetry. The Lorentz symmetry requires that space and time are symmetric, which guarantees that frequency must scale the same way as wavevector: $\omega_{k} \sim k$; this implies $z=1$.

The presence of fermions introduces additional gapless fermionic excitations, which can potentially change the critical properties of these two universality classes. We find that in the presence of fermions, the coupling between bosons and gapless fermionic excitations near the Fermi surface leads to singular terms in the effective field theory for SIT. Such singular terms turn out to be irrelevant at the $z=2$ fixed point, thus this fixed point is stable. On the other hand they lead to runaway renormalization group ( $\mathrm{RG}$ ) flows at the $z=1$ fixed point, thus destabilizing this fixed point; as a result near the tips of the Mott insulator lobes SIT either becomes a fluctuation-driven first order transition, or becomes a different 2 nd order transition. In next section we will also study the pairing interaction between fermions mediated by quantum critical fluctuations near SIT, and show that under certain conditions the fermion pairing gap and transition temperature peaks near SIT.

We begin our discussion with case (i) of Bose-Fermi

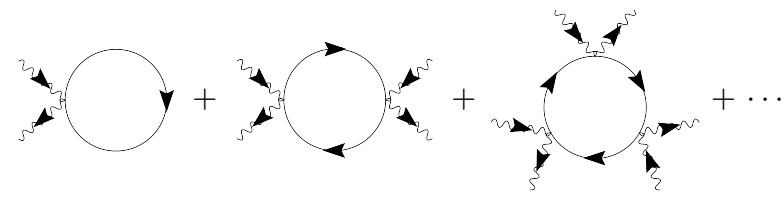

FIG. 2: Feynman diagrams representing terms in effective bosonic action generated from integrating out fermions, for case (i) of Bose-Fermi mixture. The solid and wavy lines are fermion and boson propagators respectively.

mixture, described by the following Hamiltonian:

$H=H_{B}-t_{f} \sum_{<i j>}\left(f_{i}^{\dagger} f_{j}+\right.$ h.c. $)-\mu_{f} \sum_{i} n_{f i}+V \sum_{i} n_{b i} n_{f i}$,

where $f_{i}$ is the fermion operator on site $i, n_{f i}=f_{i}^{\dagger} f_{i}$, and $V$ is the Bose-Fermi interaction strength. Even though the Hamiltonian (2) is still quite simple, it actually has a very rich phase diagram [5]. To avoid complications associated with instabilities like phase separation or bound state formation, we assume $V$ to be weak and fermion density to be low. To derive the effective field theory for boson SIT, we use a functional integral representation of Eq. (2), and integrate out the (quadratic) fermionic degrees of freedom to obtain an effective action in terms of the bosons only. This procedure generates additional interaction terms for the bosons, in the form of

$\sum_{n} \Lambda_{2 n}\left(q_{1}, \cdots, q_{n} ; q_{1}^{\prime}, \cdots, q_{n}^{\prime}\right) \bar{b}\left(q_{1}\right) \cdots \bar{b}\left(q_{n}\right) b\left(q_{1}^{\prime}\right) \cdots b\left(q_{n}^{\prime}\right)$.

Here $q=(\mathbf{q}, \omega)$ includes both momentum and frequency, and $b$ is the bosonic field of the effective field theory. These terms are represented diagramatically in Fig. 2.

Because there exist gapless excitations around the Fermi surface, tracing out fermions leads to terms in (3) with singular coupling functions $\Lambda_{2 n}$ at long wavelength and/or low frequency (i.e., $\Lambda_{2 n}(q)$ is non-analytic at small $\mathbf{q}$ and/or $\omega$ ). The most studied cases with such singularities generated by tracing out gapless fermions are Hertz theories for magnetic transitions in metals [6], where due to the direct coupling between order parameter and fermions, singularities appear at the quadratic level in the form of Landau damping. Such singularity not only changes the critical properties of the phase transition at Gaussian (or tree) level[ [6], but also casts the validity of integrating out fermions in doubt [7].

In the present case however, we find the singularities are less severe. This is because here the order parameter $b$ does not couple to fermions directly; instead the coupling is in the form of density-density interaction between bosons and fermions. As a result no singularity appears in the quadratic action (i.e., no singularity in $\Lambda_{2}(q)$ ); the bosons only receive a Hartree shift of chemical potential here. Consequently power counting and critical behavior do not change at this level. 
Singularities do appear in higher order terms of (3), and the leading singularity is in a quartic term of the form:

$$
\left.\left.\lambda_{4} \int d \mathbf{q} d \omega \frac{|\omega|}{|\mathbf{q}|}|| b\right|^{2}(\mathbf{q}, \omega)\right|^{2},
$$

where $|b|^{2}(\mathbf{q}, \omega)$ is the Fourier transform of $|b(\mathbf{r}, \tau)|^{2}$. [8] The importance of this singular quartic term depends on the dynamical exponent $z$. For $z=2, \omega$ scales as $|\mathbf{q}|^{2}$; it is clear that it is much less important than the regular quartic term $\int d \mathbf{r} d \tau|b(\mathbf{r}, \tau)|^{4}$, and in fact scales to zero from a simple power counting analysis which leads to the RG flow for $\lambda_{4}$ :

$$
\frac{d \lambda_{4}}{d \log s}=(5-d-2 z) \lambda_{4},
$$

where $s$ is the length rescaling factor that relates the length before and after an RG transformation: $x^{\prime}=x / s$ (the corresponding transformation for time is $t^{\prime}=t / s^{z}$, which provides the technical definition of dynamical exponent $z$ ). Clearly $\lambda_{4}$ is an irrelevant coupling for $z=2$ and $d>1$. We thus find that the $z=2$ quantum critical point (QCP) of the SIT is stable against weak Bose-Fermi coupling in this case.

The situation is very different at the particle-hole symmetric QCP of SIT with $z=1$. In this case Eq. (5) suggests $\lambda_{4}$ is marginal for $d=3$ while relevant for $d=2$; it can thus change the critical properties of the SIT at this particle-hole symmetric QCP. Sachdev and Morinari [9] analyzed a very similar model with the same type of singular term in a different context. As they showed, the $\lambda_{4}$ coupling is actually non-renormalizable, as at each step of RG, new relevant or marginal couplings of different types of singularities appear, as a result of which the standard RG procedure is unstable. As an alternative they introduced a new field to decouple the singular coupling (4), and corresponding coupling constants; the theory becomes renormalizable after such a modification. Within a perturbative RG treatment they find that the field theory yields no fixed point at weak coupling.

There are two possible interpretations of the absence of fixed point within perturbative RG for the particle-hole symmetric case. It could indicate that the SIT is driven first order by fluctuations due to Bose-Fermi coupling around the tips of the Mott insulator lobes, as indicated in Fig. 1b. Another more interesting possibility is that there actually exists a strong-coupling fixed point that is inaccessible to perturbative RG. If this turns out to be the case the SIT would remain 2nd order, but the critical properties would be controlled by the new fixed point at which the Bose-Fermi coupling strength flows to a universal value; this would dramatically affect the fermion properties at the transition (to be discussed later). In this case the phase diagram remains that of Fig. 1a, but the critical behavior at the tips is no longer that of $d+1$ dimensional XY model. Unfortunately the tree-level RG equation (5) as well as the more elaborate treatment that includes loop contributions of Ref. 9 cannot resolve which of the two scenarios is realized. This is because the perturbative RG flow equation (5) is well controlled only at weak coupling or small $\lambda_{4}$. While it clearly establishes the instability of the $d+1$ dimensional XY fixed point $(z=1)$ when the singular coupling $\lambda_{4}$ (due to presence of fermions) is present, one can not tell where the RG flow brings the system to, unless a more stable fixed point (with only one relevant perturbation) is found at weak coupling. On the other hand it would be very interesting to use numerical methods like quantum Monte Carlo to resolve which of the two scenario is realized; in such studies particle-hole symmetry can be ensured by fixing the boson number while varying $U / t_{b}[10]$.

We now discuss case (ii) of Bose-Fermi mixture, in which the bosons are molecules made of two fermions with opposite spins, with density imbalance between them. Recently, much interest has focused on the problem of pairing in such imbalanced fermionic systems 11, 12, 13], with the hope of realizing novel superfluid phases different from the BCS state [14]. In the present paper we consider the relatively simple regime of strong pairing (or BEC) regime, in which one can think that all the minority (-) fermions are paired with majority $(+)$ fermions to form bosonic molecules, while the unpaired + fermions form a Fermi gas; phase separation does not occur when the imbalance is small in this regime 15]. As a result bosonic molecules coexist with unpaired fermionic atoms. The simplest model that contains the basic physics is the two-channel model on a lattice with one band for both the bosons and fermions:

$$
\begin{aligned}
H & =H_{B}-t_{f} \sum_{<i j>, \sigma}\left(f_{\sigma i}^{\dagger} f_{\sigma j}+h . c .\right)-\sum_{i \sigma}(\mu+\sigma h) n_{\sigma i} \\
& -g \sum_{i}\left(f_{+i}^{\dagger} f_{-i}^{\dagger} b_{i}+h . c .\right) .
\end{aligned}
$$

Here $\sigma= \pm 1$ is the (pseudo)spin index for the fermions, $f_{\sigma i}$ is annihilation operator of the fermion on site $i$ with spin $\sigma$ and $n_{\sigma i}=f_{\sigma i}^{\dagger} f_{\sigma i}$. The fermion species are at chemical potentials $\mu_{f \sigma}=\mu+\sigma h$, while the boson is at chemical potential $\mu_{b}=2 \mu-\nu$. Here $h$ is the "Zeeman field" which imbalances the fermion densities, and $\nu$ is the "detuning" across the Feshbach resonance which scans between the BCS and BEC limits. As mentioned earlier, we focus mainly in the regime of strong pairing or large negative detuning, $|\nu| \gg g, \mu, t, U$. In this regime fermions with opposite spins form closely bound molecules, and the system can sustain considerable spin imbalance without phase separation 15. The unpaired majority $(+)$ fermions form a single Fermi surface 15, 16 with a volume satisfying a generalized Luttinger theorem 17]. It should be noted that while we have three types of particles, due to the $g$ term in (6) there 


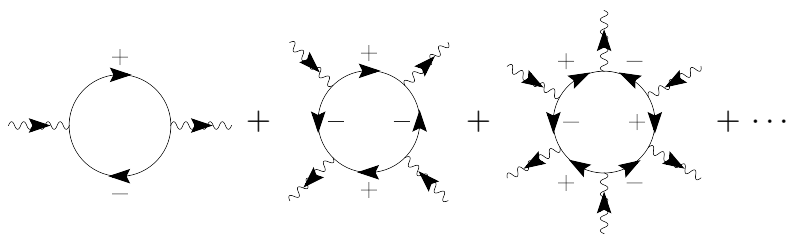

FIG. 3: Same as Fig. 2, for case (ii) of Bose-Fermi mixture.

are only two conserved charges: $N_{\sigma}=\sum_{i}\left(b_{i}^{\dagger} b_{i}+f_{\sigma i}^{\dagger} f_{\sigma i}\right)$. The boson superfluid and insulating phases here are defined with respect to the transport of conserved charge $N_{-}$. Physically this reflects the fact that at low energy minority (-) fermions exist in the form of constituents of bosonic molecules at low-energy.

We can again trace out the fermions in Eq. (6), which generates effective boson interaction terms of the form (3) that are represented by a new set of diagrams of Fig. 3. While the Hamiltonian (6) and the Feynman diagrams Fig. 3 look very different from those of the previous case, and in particular the superfluid order parameter $(b)$ couples directly to fermions here, the singularities generated by tracing out fermions turn out to be identical. Heuristically it can be seen from the following considerations. In the Hamiltonian (6) the majority $(+)$ fermions are gappless but the minority (-) fermions remain fully gapped; as a result the - fermion Green's function has no pole at low-energy and can be treated as a number $(-1 /|\mu-h|)$. Diagramatically, one can thus shrink the - fermion lines to a point for the diagrams in Fig. 3, which reduces them to the same diagrams as those in Fig. 2. Alternatively, one can also see this by first integrating out the fully gapped - fermions; this generates a quartic (or density-density) interaction between bosons and gapless + fermions of the same form as in (2). They thus have the same singularities, and we conclude that these two cases of Bose-Fermi mixtures share the same phase diagram and critical properties of SIT.

\section{FERMION PAIRING NEAR BOSON SUPERFLUID-INSULATOR TRANSITION}

In this section we turn our discussion to the behavior of fermions, especially near SIT. It should be clear from the discussion above that the fermion physics is also similar for cases (i) and (ii), and we focus on case (i) below. It has been known for a long time 18] that boson density fluctuations mediate an attractive interaction for case (i) of Bose-Fermi mixture, which at weak coupling takes the form [19, 20]

$$
V_{e f f}(\mathbf{q}, \omega)=V^{2} \chi(\mathbf{q}, \omega),
$$

where $\chi(\mathbf{q}, \omega)$ is the boson density susceptibility. This attarctive interaction leads to fermion pairing, an effect that has been studied in some detail for the case that the bosons are deep in the superfluid phase 19, 20, 21, 22]; in this case the effective interaction is dominated by single phonon exchange, and takes a form very similar to the electron-phonon interaction in metals. When the bosons are in the Mott insulator phase $\chi(\mathbf{q}, \omega)$ is zero at $T=0$ and small $\omega$, and takes an activated behavior at low $T$ due to the Mott-Hubbard gap; as a result the effective pairing interaction is extremely weak. In the following we study the pairing physics near the particle-hole asymmetric SIT with $z=2$, where the critical properties are known and the Bose-Fermi interaction can be treated perturbatively due to its irrelevance in the RG sense. We will comment on the case of particle-hole symmetric SIT later on.

The critical behavior of particle-hole asymmetric SIT is that of the dilute Bose gas transition 24. For $d=2$ or 3 , the theory is at or above its upper critical dimension, and a mean-field (or Hartree-Fock) treatment of the boson interaction $U$ in the calculation of $\chi(\mathbf{q}, \omega)$ in Eq. (7) is justifiable even near the transition. For simplicity we focus on $d=3$ below to avoid complications associated with logarithmic correction at the upper critical dimension $d=2$. For weak pairing (which is assumed here), we may neglect the frequency dependence in Eq. (7) and reduce it to a static attractive interaction:

$$
V_{e f f}(\mathbf{q})=-\frac{V^{2} \kappa}{1+|\mathbf{q}|^{2} \xi^{2}}
$$

where $\kappa=\frac{\partial n_{B}}{\partial \mu_{B}}$ is the boson static compressibility, and $\xi$ is proportional to the boson superfluid correlation length (with an $O(1)$ prefactor). This attractive interaction leads to fermion superfluid transition temperature in angular momentum channel $l$ :

$$
T_{c}^{F}=A_{l} E_{F} \exp \left\{-1 /\left[D\left(E_{F}\right) \Gamma_{l}\right]\right\},
$$

where $E_{F}$ is Fermi energy, $D\left(E_{F}\right)$ is fermion density of states at $E_{F}, A_{l}$ is a constant of order 1 (e.g., $A_{l=0} \approx$ $0.61)$, and

$$
\Gamma_{l}=\frac{2 l+1}{2} \int_{0}^{2 \pi} d \cos \theta V_{e f f}\left[2 k_{F} \sin (\theta / 2)\right] P_{l}(\cos \theta)
$$

is the pairing interaction in angular momentum channel $l$. Here $k_{F}$ is the Fermi wave vector and $P_{l}$ is the Legendre polynomial.

On the superfluid side with $\Delta \mu>0(\Delta \mu$ is the difference between $\mu_{B}$ and the phase boundary) and below the boson superfluid transition temperature $T_{c}^{B}$, straightforward calculation shows [23]

$$
\kappa \approx 1 / U
$$

which is mostly independent of $\Delta \mu$, and

$$
\xi \sim \sqrt{t_{b} / \Delta \mu}
$$

which diverges with mean-field exponent as $\Delta \mu \rightarrow 0$. The increasing correlation length $\xi$ reduces the $s$-wave pairing 
strength $\Gamma_{0}$. However it enhances the $p$-wave pairing [21] strength $\Gamma_{1}$ due to the stronger momentum dependence of the pairing interaction, as long as $\xi \lesssim 1 / k_{F}$. Since for the single component fermion in our model (2) $s$-wave pairing is not allowed and the leading pairing instability is in the $p$-wave channel, we expect fermion gap and $T_{c}^{F}$ to increase as one approaches SIT; thus one expects $T_{c}^{F}$ to peak very close to the critical point with $\Delta \mu=0$.

Since $T_{c}^{B} \rightarrow 0$ as $\Delta \mu \rightarrow 0, T_{c}^{F}$ will cross $T_{c}^{B}$ when one gets sufficiently close to the SIT. In this case the pairing interaction is controlled by the finite $T$ properties of the $z=2 \mathrm{QCP}$ and becomes strongly $T$-dependent. Again using mean-field approximation we obtain

$$
\kappa \sim 1 /\left(U^{2} t_{B} T\right)^{1 / 4}
$$

and 24]

$$
\xi \sim\left[t_{b}^{5} /\left(U^{2} T^{3}\right)\right]^{1 / 4} .
$$

In this case $T_{c}^{F}$ needs to be determined self-consistently by solving Eq. (9). Our qualitative results on fermion pairing are summarized in Fig. 4. 25]

As discussed earlier, Bose-Fermi interaction is relevant at the particle-hole symmetric QCP of SIT, and may flow to a strong coupling fixed point. If this turns out to be the case then fermions have a universal strong pairing interaction at this QCP, a situation very similar to Feshbach resonance. We thus expect $T_{c}^{F} \sim \tilde{E_{F}}$, where $\tilde{E_{F}}$ is the energy scale at which the Bose-Fermi interaction has flown to the universal value. We expect $\tilde{E_{F}} \sim E_{F}$ for $V \sim E_{F}$, as there is no other energy scale for the fermions in this case. This could be an alternative way to achieve "high" $p$-wave $T_{c}^{F}$. In both the speculative case here and the concrete case of particle-hole asymmetric QCP with $z=2$ discussed above, we find $p$-wave $T_{c}^{F}$ peaks at the QCP due to pairing interaction mediated by quantum critical fluctuations. This is similar to the quantum criticality scenario of high $T_{c}$ superconductivity in cuprates, and provides a concrete example of this scenario.

\section{CONCLUDING REMARKS}

Bose-Fermi mixtures represent new quantum manybody systems realized in trapped cold atomic gases, that do not have straightforward correspondences in electronic condensed matter systems. In the latter case the constituent particles, namely electrons, are fermions. When the electrons form Cooper pairs, the system may also be described (at low energy) using boson only models like the Bose Hubbard model; in these cases typically the fermionic degrees of freedom are fully gapped and do not enter the low-energy effective theory.

In the present paper we have explored some new physics in Bose-Fermi mixtures, in which low-energy bosonic and fermionic excitations co-exist and interact

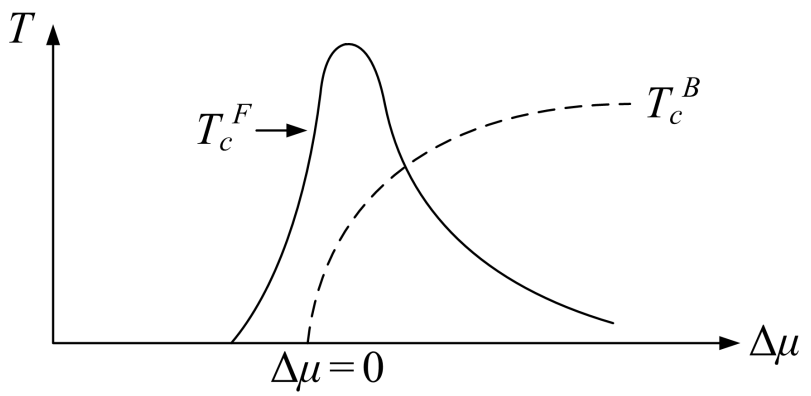

FIG. 4: Finite temperature phase diagram near the particlehole asymmetric boson superfluid-insulator transition, at $\Delta \mu=0$ and $T=0$. The solid line is $p$-wave fermion superfluid transition temperature, while dashed line is boson superfluid transition temperature.

with each other. More specifically, we have studied the effect of fermions on the critical properties of boson superfluid-insulator transition (SIT), and the pairing interaction between fermions mediated by quantum critical fluctuations near SIT. In the latter case we predict the fermion pairing gap and superfluid transition temperature peak near boson SIT. It would be very interesting to use quantum Monte Carlo or other numerical methods to test the predictions of this paper, especially to resolve the nature of the particle-hole symmetric boson SIT in the presence of fermions.

The author thanks Subir Sachdev and Fei Zhou for helpful conversations, and Q. Cui for graphics assistance. Parts of this work were performed at Kavli Institute for Theoretical Physics (KITP, which was supported in part by the National Science Foundation under Grant No. PHY05-51164), and Kavli Institute for Theoretical Physics China (KITPC). This work was supported in by National Science Foundation grants No. DMR-0225698 and No. DMR-0704133.

[1] M. Greiner, O. Mandel, T. Esslinger, T. W. Hänsch, and I. Bloch, Nature 415, 39 (2002).

[2] For a review, see Immanuel Bloch, Jean Dalibard, and Wilhelm Zwerger, arXiv:0704.3011.

[3] Matthew P. A. Fisher, Peter B. Weichman, G. Grinstein, and Daniel S. Fisher, Phys. Rev. B 40546 (1989).

[4] For a review, see S. Sachdev, Quantum Phase Transitions, Cambridge University Press, Cambridge, 1999.

[5] M. Lewenstein, L. Santos, M. A. Baranov, and H. Fehrmann, Phys. Rev. Lett. 92, 050401 (2004).

[6] J. A. Hertz, Phys. Rev. B 14, 1165 (1976).

[7] In fact the validity of the Hertz approach has been a subject of intense debate recently; for a recent review of the current status, see D. Belitz, T. R. Kirkpatrick, and Thomas Vojta, Rev. Mod. Phys. 77, 579 (2005).

[8] In fact this is the same type of singularity encountered in the Hertz theory in its quadratic action. The source of 
the difference is that fermion particle-hole pairs couple to the order parameter in the Hertz theory, while here it couples to the square of the order parameter. More generally, there is a one-to-one correspondence between Feynman diagrams in Hertz theory and those encoutered here (see Fig. 2); a diagram with $n$ external legs in Hertz theory correspnds to a diagram with $2 n$ legs here. The doubling of external legs leads to doubling of power of boson fields in the corresponding term of effective field theory, significantly reducing its importance; the bigger $n$ is, the less important it becomes compared to the corresponding therm in Hertz theory.

[9] S. Sachdev and T. Morinari, Phys. Rev. B 66235117 (2002).

[10] Simulations on one-dimensional Bose-Fermi mixtures have been performed very recently; see Lode Pollet, Matthias Troyer, Kris Van Houcke, and Stefan M. A. Rombouts, Phys. Rev. Lett. 96, 190402 (2006); Lode Pollet, Corinna Kollath, Ulrich Schollwoeck, and Matthias Troyer, arXiv:cond-mat/0609604 F. Hebert, F. Haudin, L. Pollet, and G.G. Batrouni, arXiv:0706.3799 X. Barillier-Pertuisel, S. Pittel, L.Pollet, and P. Schuck, arXiv:0706.2133. The physics discussed in the present paper applies to 2- and 3-dimensional systems, but not to one-dimensional systems. Simulating higher dimensional systems will be more challenging, but also with bigger pay-off.

[11] Pairing in Fermionic Systems: Basic Concepts and Modern Applications, Mark Alford, John Clark and Armen Sedrakian, eds, World Scientific (2006).

[12] M. W. Zwierlein, A. Schirotzek, C. H. Schunck, and W. Ketterle, Science 311, 492 (2006).

[13] G. B. Partridge, W. Li, R. I. Kamar, Y. Liao, and R. G. Hulet, Science 311, 503 (2006).
[14] For a review, see K. Yang, in Ref. 11 (cond-mat/0603190).

[15] D. E. Sheehy and L. Radzihovsky, Phys. Rev. Lett. 96, 060401 (2006); Annals of Physics 322, 1790 (2007).

[16] K. Yang, cond-mat/0508484.

[17] S. Sachdev and K. Yang, Phys. Rev. B 73, 207 (2006).

[18] J. Bardeen, G. Baym and D. Pines, Phys. Rev. 156, 174504 (1967).

[19] M. J. Bijlsma, B. A. Heringa, and H. T. C. Stoof, Phys. Rev. A 61, 053601 (2000).

[20] L. Viverit, C. J. Pethick, and H. Smith, Phys. Rev. A 61, 053605 (2000).

[21] D. V. Efremov and L. Viverit, Phys. Rev. B 65, 134519 (2002).

[22] For more recent work, see, e.g., L. Viverit, Phys. Rev. A 66, 023605 (2002); F. Matera, Phys. Rev. A 68, 043624 (2003); D.-W. Wang, M. D. Lukin and E. Demler, Phys. Rev. A 72, 051604 (2005); D.-W. Wang, Phys. Rev. Lett. 96, 140404 (2006); F. D. Klironomos and S.-W. Tsai, arXiv:cond-mat/0702660.

[23] In this case the additional particles or holes (measured from the fixed boson density of the nearby Mott phase) form a dilute Bose gas, and we can use the results known about that system; see, e.g. Eq. (17) of Ref. 20.

[24] See Ch. 11 of Ref. 4.

[25] While $T_{c}^{F}$ is determined by Eq. (9), one needs knowledge of $\kappa$ and $\xi$ as input to obtain its quantitaive value. Using the known critical properties of $z=2$ SIT we have obtained the exact scaling behavior of $\kappa$ and $\xi$ in Eqs. (11]1213/14), but we cannot determine their nonuniversal prefactors which depend on microscopic details. Thus our results on $T_{c}^{F}$ is only qualitative. However the conclusion that it peaks near SIT is robust. 